
\title{
3 Research Square \\ Inhibitory Effects of LOXL2 Knockdown On Cellular Functions of Liver Cancer Stem Cells
}

\section{$\mathrm{Na} \mathrm{Li}$}

The First Affiliated Hospital of Dalian Medical University

\section{Huan Gu}

The First Affiliated Hospital of Dalian Medical University

\section{Liu Liu}

The First Affiliated Hospital of Dalian Medical University

\section{Xiao Li Zhang}

The First Affiliated Hospital of Dalian Medical University

\section{Qiu Luo Cheng}

The First Affiliated Hospital of Dalian Medical University

\section{Ying Zhu ( $\nabla$ zhuyingsh52@126.com )}

The First Affiliated Hospital of Dalian Medical University https://orcid.org/0000-0002-0624-7974

\section{Research Article}

Keywords: Hepatocellular carcinoma, Lysyl oxidase-like 2, Liver cancer stem cells, Invasion, Migration, Apoptosis

Posted Date: December 10th, 2021

DOI: https://doi.org/10.21203/rs.3.rs-1147621/v1

License: (c) (i) This work is licensed under a Creative Commons Attribution 4.0 International License. Read Full License 


\section{Abstract}

Background and aim: Lysyl oxidase-like 2 (LOXL2) plays a role in tumor microenvironment formation and metastasis of hepatocellular carcinoma (HCC), which has a high mortality burden. Liver cancer stem cells (LCSCs) are related with the major malignant phenotypes of HCC. The function of LOXL2 in regulation of LCSCs remains unknown.

Methods: $\mathrm{CD} 133^{+} \mathrm{HepG} 2$ and CD133 ${ }^{+} \mathrm{Hep} 3 \mathrm{~B}$ cells were sorted by fluorescence-activated cell sorting (FACS) from two human hepatoblastoma cell lines. Spheroid formation, apoptosis, cell cycle, as well as transwell assays were performed upon LOXL2 knock down in CD133+HepG2 and CD133 ${ }^{+} \mathrm{Hep} 3 \mathrm{~B}$ cells. Protein and mRNA levels were quantified by Western blotting, Immunofluorescence and real-time PCR.

Results: Knockdown of $L O X L 2$ decreased spheroid formation, migration and invasion $(p<0.05)$, also induced apoptosis $(p<0.05)$ and cell cycle arrest $(p<0.05)$ in CD133 ${ }^{+}$HepG2 and CD133 $3^{+} H e p 3 B$ cells. Knockdown of $L O X L 2$ effectively inhibited expression of the anti-apoptosis proteins baculoviral IAP repeat-containing 3 (BIRC3) and murine double minute 2 (MDM2) $(p<0.01)$, as well as autophagy marker microtubule-associated protein 1 light chain 3 B (LC3) and autophagy gene ATG5 in CD $133^{+}$HepG 2 and CD133+Hep3B cells $(p<0.01)$.

Conclusions: The results revealed that LOXL2 inhibition could reduce the proliferation and expansion of LCSCs, making LOXL2 inhibitors an attractive and novel therapeutic strategy of HCC.

\section{Introduction}

Hepatocellular carcinoma (HCC) is very common and has a high mortality burden ${ }^{[1,2]}$. Hepatitis B and C, metabolic dysfunction associated fatty liver disease and alcohol intake are the main risk factors of HCC $[2,3]$. Hepatocarcinogenesis is accompanied by resistance to cellular death signals, metabolic changes, and a failure to clear damaged precancerous hepatocytes by the immune system ${ }^{[4]}$. The poor prognosis and response to therapy of $\mathrm{HCC}$ is in part due to the presence of CSCs, an immune-privileged cell population that can evade immune surveillance more effectively than non-CSCs ${ }^{[5,6]}$. Liver cancer stem cells (LCSCs) are a subset of hepatocarcinoma cells with pluripotent and self-renew properties related to the major malignant phenotypes of $\mathrm{HCC}{ }^{[3,7,8]}$. The expression of CSC-related genes such as NANOG, SOX2 and OCT4 can be promoted by oncogenes and/or overexpression of oncoproteins such as the HBV envelope protein PreS1, thereby driving the malignant biological behaviors of LCSCs ${ }^{[9]}$. LCSCs can be characterized through cell surface markers, such as CD133 ${ }^{[10,11]}$. CD133 is a transmembrane cell surface single chain glycoprotein, which is the original marker of hematopoietic stem cells, neural stem cells and liver stem / progenitor cells. It is an important indicator of malignant progression, patient survival and recurrence rate. The expression of CD133 in tumor cells is related to the stem cell-like characteristics ${ }^{[12 ~ 14]}$. 
Lysyl oxidase-like 2 (LOXL2) belongs to the lysyl oxidase (LOX) family, members of which are implicated in diverse pathophysiology including developmental regulation, cell motility, cellular senescence, and tumor suppression or promotion ${ }^{[15 ~ 17]}$. The roles played by LOXL2 in fibrosis, tumorigenesis, and metastasis have been extensively reported, and it has also been recognized as a therapeutic target ${ }^{[16,18 \text {, }}$ 19]. Overexpression of LOXL2 is related with poor prognosis of patients with malignant tumors, such as colon cancer, esophageal cancer, and squamous cell carcinoma ${ }^{[15,20,21]}$. LOXL2 can induce epithelial to mesenchymal transition (EMT), its overexpression negatively affects the clinicopathological features of different tumor types ${ }^{[22]}$. In HCC, LOXL2 is upregulated and plays a key role in HCC metastasis by mediating EMT and extracellular matrix remodeling, subsequently enhancing HCC invasion ${ }^{[22,23]}$. LOXL2 is also crucial in tumor microenvironment formation and metastatic niche in $\operatorname{HCC}{ }^{[23,24]}$. However, the functions and regulatory mechanism of LOXL2 in LCSCs remains unknown.

In this study, we sorted the $\mathrm{CD} 133^{+} \mathrm{HepG} 2$ and $\mathrm{CD} 133^{+} \mathrm{Hep} 3 \mathrm{~B}$ cells from two human hepatoblastoma cell lines. The cellular function of the two cell lines, including proliferation, migration, invasion as well as apoptosis and cell cycle assay, were demonstrated upon LOXL2 gene knock down. To understand whether LOXL2 benefited LCSCs from escaping apoptotic cell death, we investigated the change of gene expression of anti-apoptosis proteins baculoviral IAP repeat-containing 3 (BIRC3, also known as cIAP2) and murine double minute 2 (MDM2) in CD133+HepG2 and CD133 ${ }^{+}$Hep3B cells upon LOXL2 knockdown. Moreover, to explore whether LOXL2 was related with autophagic cell death, we investigated the expression change of autophagy marker microtubule-associated protein 1 light chain 3 (LC3) B and autophagy gene ATG5 in CD133+HepG2 and CD133+Hep3B cells upon LOXL2 gene knock down.

\section{Materials And Methods}

\section{Cell lines}

The human hepatoblastoma cell lines HepG2 and Hep3B were purchased from Chinese Academy of Sciences (Shanghai, China). Cells were cultured in RPMI 1640 medium (Gibco, Thermo Fisher Scientific, Waltham, MA, USA) with $10 \%$ fetal bovine serum (FBS; PAN, Germany) and $1 \%$ penicillin and streptomycin (Gibco) at $37.0^{\circ} \mathrm{C}$ in $5 \% \mathrm{CO}_{2}$.

\section{Isolation of LCSCs through fluorescence activated cell sorting (FACS)}

HepG2 or Hep3B cells were resuspended in phosphate-buffered saline (PBS), then incubated with FcR blocking reagent (Miltenyi Biotec, Bergisch Gladbac, Germany) and phycoerythrin (PE)-conjugated CD133 antibodies (Miltenyi Biotec) at $4^{\circ} \mathrm{C}$ for 20 min before sorting by FACS on a BD Influx (BD Biosciences, Franklin Lakes, $\mathrm{NJ}, \mathrm{USA}$ ). About $60 \%$ of $\mathrm{CD} 133^{+}$cells were sorted based on strong positivity. Isotypematched mouse IgG was used to exclude non-specifically-stained cells. CD133+HepG2 and CD133 ${ }^{+} \mathrm{Hep3B}$ cells were cultured in DMEM/F12 (HyClone, Logan, UT, USA) with $20 \mathrm{ng} / \mathrm{ml}$ recombinant 
epidermal growth factor (EGF; PeproTech, Rocky Hill, NJ, USA), $20 \mathrm{ng} / \mathrm{ml}$ basic fibroblast growth factor (bFGF; PeproTech) and 2\% B27 supplement (Gibco) at $37.0^{\circ} \mathrm{C}$ in $5 \% \mathrm{CO}_{2}$. The $\mathrm{CD} 133$ positive proportion of cells was detected by flow cytometry.

\section{In vitro LOXL2 gene silencing}

LOXL2 gene in $\mathrm{CD} 133^{+} \mathrm{HepG} 2$ cells and $\mathrm{CD} 133^{+} \mathrm{Hep} 3 \mathrm{~B}$ cells were stable knockout using siRNA lentiviral vector (LV; GeneChem, Shanghai, China), the control group were transfected with siRNA negative control lentiviral vector. The vectors were encoded with green fluorescent protein (GFP). Cells were transduced with 25 multiplicity of infection (MOI) lentiviral particles, and HiTransG P (GeneChem, Shanghai, China) was used to promote transfer efficiency. After 48 to 72 hours, the effect of cell infection was observed under fluorescence microscope to make sure that more than $80 \%$ of cells show GFP. Cells were transduced for $72 \mathrm{~h}$ before treated with $2 \mu \mathrm{g} / \mathrm{mL}$ puromycin for 2 weeks. Transfection efficiency was confirmed by RT-PCR and western blotting.

After stable knockout of LOXL2 by transfected with LOXL2 siRNA lentivirus, the CD133 ${ }^{+} \mathrm{Hep} 3 \mathrm{~B}$ cell line presented with growth retardation, even died. $C D 133^{+} \mathrm{Hep} 3 \mathrm{~B}$ cell line was more susceptible to LOXL2 gene deletion. Thus, we adjusted to transient transfection of $\mathrm{CD} 133^{+} \mathrm{Hep3B}$ cell line by siRNA in the follow-up study after spheroid formation assay. Pre-validated Silencer ${ }^{\circledR}$ select siRNAs against $\angle O X L 2$, negative control siRNA, and GAPDH siRNA (Thermo Fisher Scientific) were transfected respectively into $5 \times 10^{5}$ $\mathrm{CD}^{133^{+}} \mathrm{Hep3B}$ cells at a dose of 25 pmol in six-well plates using the Lipofectamine RNAiMAX ${ }^{\mathrm{TM}}$ transfection reagent (Invitrogen, Waltham, MA, USA) for $24 \mathrm{~h}$.

\section{Quantitative real-time PCR}

Total RNA was extracted from CD133+HepG2 cells and CD133+Hep3B cells. Then, cDNA was synthetized by reverse transcription with the Evo M-MLV RT Kit for qPCR (AG Bio, China). PCR was performed by Applied Biosystems 7500 PCR System (Thermo Fisher Scientific) with SYBR® Green Premix Pro Taq HS qPCR Kit (AG Bio). GAPDH was taken as the internal control. The amplification conditions were as follows: pre-denaturation $\left(95^{\circ} \mathrm{C}, 30 \mathrm{~s}\right)$, denaturation $\left(95^{\circ} \mathrm{C}, 5 \mathrm{~s}\right)$, annealing and extension $\left(60^{\circ} \mathrm{C}, 30 \mathrm{~s}\right)$, all for a total 40 cycles. The experimental Ct values were normalized against GAPDH. $2^{-\triangle \triangle C T}$ method was used to analyze the data.

\section{Western blotting}

Total protein lysate was extracted on ice using radioimmunoprecipitation assay (RIPA) buffer, phenylmethanesulfonyl fluoride, phosphatase inhibitor, and protease inhibitor (KeyGEN, Nanjing, China). After separated in SDS-PAGE, proteins were transferred to polyvinylidene difluoride (PVDF) membrane. Then, the PVDF membrane was blocked with $5 \%$ buffer bovine serum albumin in TBST and exposed to primary antibody at $4^{\circ} \mathrm{C}$ overnight. Next, the PVDF membrane was washed in TBST (10 min $\times 3$ times) and exposed to secondary antibody (1:5000) at room temperature for 1-1.5 $\mathrm{h}$. The band was incubated in BeyoECL Plus (Beyotime China) and detected by chemiluminescence. 


\section{Spheroid formation}

Cells were collected and washed with PBS. After ACCUTASE (Sigma-Aldrichl, USA) digestion, single cells $\left(1 \times 10^{4}\right)$ were seeded in ultra-low adhesion 6-well plate (Crystalgen, Commack, NY, USA) containing serum-free culture (SFC) medium, which composed of DMEM/F12 (Hyclone), 20 ng/ml EGF (PeproTech), $20 \mathrm{ng} / \mathrm{ml} \mathrm{bFGF}$ (PeproTech), and 2\% B27 supplement (Life Technologies). Add $1 \mathrm{ml}$ of fresh SFC into the cells twice a week. After culturing for 10-15days, the number of tumor spheroids $(>50 \mu \mathrm{m})$ were determined.

\section{Flow cytometric apoptosis analysis}

Cells in logarithmic growth phase were collected and digested into single ones by ACCUTASE (SigmaAldrichl). $1 \times 10^{5} \sim 1 \times 10^{6}$ single cells were washed with cold PBS and $1 \times$ binding buffer, respectively. Next, cells were resuspended in $100 \mu \mathrm{L}$ binding buffer with $5 \mu \mathrm{L}$ Annexin V-FITC and $10 \mu \mathrm{L}$ PI staining solution, and incubated in the dark for $15 \mathrm{~min}$ at room temperature. Then, apoptosis was analyzed with a FACS Calibur instrument.

\section{Flow cytometry cell cycle analysis}

Cells were collected and digested into single ones by ACCUTASE (Sigma-Aldrichl). Then, the cells were washed with cold PBS, and fixed with $70 \%$ ethanol at $4^{\circ} \mathrm{C}$ overnight. After being washed, cells were incubated with $2 \mathrm{~mL}$ staining solution (propidium iodide) in the dark for $30 \mathrm{~min}$ at room temperature. Then, cell cycle was analyzed with a FACSCalibur instrument (BD Biosciences).

\section{Transwell assay for migration and invasion}

Cells were starved for 24 hours, and suspended with RPMI 1640 medium. $5 \times 10^{4}$ cells in $200 \mu \mathrm{L}$ RPMI 1640 medium were added into the upper chambers of $8 \mu \mathrm{m}$ pore size transwells. $700 \mu \mathrm{L}$ medium with $10 \%$ FBS were added into the lower ones. For the invasion assay, the bottom of the transwell chamber was laid with diluted Matrigel (BD Biosciences). The cells in transwell were cultured at $37^{\circ} \mathrm{C}$ in $5 \% \mathrm{CO}_{2}$ for 24 hours. Next, the transwell was treated with $4 \%$ paraformaldehyde for $30 \mathrm{~min}$, and $0.1 \%$ crystal violet for $25 \mathrm{~min}$. After PBS washing, cells in the upper chambers were wiped off, and cells at the bottom of the chamber were observed under the microscope, and five fields were randomly selected for counting $(\times 100$ objective).

\section{Immunofluorescence assay}

Cells were fixed in 4\% paraformaldehyde for $30 \mathrm{~min}$ and permeabilized in $0.25 \%$ Triton X-100 for 15 min. After blocked by $5 \%$ goat serum at room temperature for $45 \mathrm{~min}$, cells were exposed to primary antibody (1:500; Abcam, Cambridge, UK) at $4^{\circ} \mathrm{C}$ overnight. Next, cells were exposed to fluorescein (FITC)conjugated goat anti-rabbit antibody (1:250; Abbkine, China) in the dark for $1 \mathrm{~h}$ at room temperature. Fluorescence images were acquired by a fluorescence microscope (Olympus, Tokyo, Japan).

\section{Statistical analysis}


Statistical analysis was performed in Prism 8 software (GraphPad Software, La Jolla, CA). Data of continuous variables are shown as mean \pm standard deviation (S.D). The difference between two independent groups was analyzed with two-sided unpaired Student's $t$-test. $p$-value $<0.05$ is regarded as statistically significant.

\section{Results}

\section{Knock down of LOXL2 impairs LCSCs spheroid formation}

To understand the effect of LOXL2 knockdown on the cellular functions of LCSCs, LOXL2 or control siRNA lentiviral vectors were transferred into $\mathrm{CD} 133^{+} \mathrm{HepG} 2$ and $\mathrm{CD} 133^{+} \mathrm{Hep} 3 \mathrm{~B}$ cell lines. Results of Western blot and PCR showed that LOXL2 was effectively knocked down in the two cell lines (Fig. 1a, b). The ability of colony formation depends on the replication immortality of tumor cells, which is one of the hallmarks of tumor ${ }^{[25]}$. To explore whether knock down of LOXL2 gene could impair tumor spheroid formation of LCSCs, CD133 ${ }^{+}$HepG 2 and $\mathrm{CD} 133^{+}$Hep3B cells were digested into single cells and cultured in SFC medium for 1-2 weeks. For $\mathrm{CD} 133^{+} \mathrm{HepG} 2$ cells, spheroid formation was significantly decreased upon silencing LOXL2 (Fig. 1c). When switching to CD133+ Hep3B cells, a more significant inhibition effect was observed (Fig. 1d). CD133 ${ }^{+}$Hep3B cell line was more susceptible to LOXL2 gene deletion. After stable knockout of LOXL2 by transfected with LOXL2 siRNA lentivirus, the growth of CD133 ${ }^{+} \mathrm{Hep} 3 \mathrm{~B}$ cell line significantly slowed down, stagnated or even died. These results indicate that LOXL2 knockdown impairs LCSCs spheroid formation.

\section{Knock down of LOXL2 inhibits LCSCs migration, and invasion}

As LOXL2 gene plays a key role in HCC metastasis, subsequently enhancing HCC invasion, we asked whether silencing LOXL2 could inhibit LCSCs expansion. The transwell migration and invasion assays were performed in CD133+HepG2 (Fig. 2a, b) and CD133+Hep3B cells (Fig. 2c, d) which subjected to siRNA-mediated LOXL2 knockdown. The migration and invasion effect of both two cell lines were decreased upon LOXL2 knockdown compared to the controls. It indicates that LOXL2 knockdown inhibits LCSCs migration, and invasion.

\section{Knock down of LOXL2 induces apoptosis and cell cycle arrest in LCSCs}

Anti-apoptosis is one of the signs of malignant tumors. We assessed apoptosis of CD133+HepG2 and CD133 ${ }^{+}$Hep3B cells by flow cytometry using Annexin V-FITC and PI. The results showed that knock down of LOXL2 significantly increased the proportion of cell apoptosis in both CD133+ HepG2 (Fig. 3a) and $\mathrm{CD}_{133^{+}}$Hep3B cells (Fig. 3b). Furthermore, we explored whether knock down of LOXL2 could also affect 
cell cycle progression in the two cell lines. We found that the proportion of CD133 ${ }^{+}$HepG 2 cell decreased in $\mathrm{G0} / \mathrm{G} 1$ phase, increased in $\mathrm{G} 2 / \mathrm{M}$ phase, and was unaltered in $\mathrm{S}$ phase upon LOXL2 knockdown (Fig. 3c). Correspondingly, the proportion of $\mathrm{CD} 133^{+} \mathrm{Hep} 3 \mathrm{~B}$ cells increased in $\mathrm{G} 0 / \mathrm{G} 1$ phase, decreased in $S$ phase, and did not change in G2/M phase (Fig. 3d). These results indicate that knock down of LOXL2 makes $\mathrm{CD}_{133^{+}} \mathrm{HepG} 2$ cell cycle arrest in G2/M phase, and CD133 ${ }^{+} \mathrm{Hep} 3 \mathrm{~B}$ cells arrest in G0/G1 phase. LOXL2 knockdown induces the apoptotic phenotype and regulates the cell cycle of LCSCs.

Knock down of LOXL2 inhibits the expression of the anti-apoptosis proteins BIRC3 and MDM2 in LCSCs

The activation of anti-apoptosis proteins benefits tumor cells from escaping apoptotic cell death ${ }^{[4,26]}$. To understand whether LOXL2 favored the survival of LCSCs by anti-apoptosis, we explored the expression of BIRC3 and MDM2 in CD133 ${ }^{+}$HepG2 and CD133+ Hep3B cells upon LOXL2 knockdown. The results showed that LOXL2 knockdown significantly decreased protein and mRNA expression of BIRC3 and MDM2 in the two cell lines compared to controls (Fig. 4a-d). Immunofluorescence analysis of CD133 ${ }^{+}$ HepG2 cells was consistent with the western blotting and RT-PCR results, with reduced level of BIRC3 and MDM2 upon LOXL2 knockdown (Fig. 4e, f). These results indicate that one of the molecular mechanisms of apoptosis induced by LOXL2 knockdown in LCSCs may lies in the downregulation of anti-apoptosis protein BIRC3 and MDM2.

Knock down of LOXL2 inhibits the expression of autophagy marker LC3B and autophagy gene ATG5 in LCSCS

As to $\mathrm{CD} 133^{+} \mathrm{Hep} 3 \mathrm{~B}$ cells proliferated significantly slow or even died after stable knockout of LOXL2, we wondered whether LOXL2 took a part in regulation of autophagic cell death of LCSCs besides antiapoptosis. So, we further explored the expression of autophagy marker LC3B and autophagy gene ATG5 in $\mathrm{CD} 133^{+} \mathrm{HepG} 2$ and $\mathrm{CD} 133^{+} \mathrm{Hep} 3 \mathrm{~B}$ cells upon LOXL2 gene knock down. The results showed that LC3B and ATG5 expression at protein and mRNA level were both significantly decreased in the two cell lines upon LOXL2 knockdown (Fig. 5a-d). Also, it was found using immunofluorescence that inhibition of LOXL2 reduced the expression of LC3B and ATG5 in CD133 ${ }^{+} \mathrm{HepG} 2$ cells. (Fig. 5e, f). These results indicate that LOXL2 knockdown inhibits the expression of autophagy marker LC3B and autophagy gene ATG5 in LCSCs.

\section{Discussion}

LCSCs, a subset of hepatocarcinoma cells with CSC-like properties, are an important therapeutic target in $\mathrm{HCC}{ }^{[3,27]}$. Here we show that knock down of $L O X L 2$ inhibits the proliferation, invasion and migration of $\mathrm{CD}_{133^{+}}$HepG2 and CD133 ${ }^{+}$Hep3B cells, and induces cell apoptosis and cell cycle arrest. This is consistent with the conclusions of Wong et al., they demonstrated that LOXL2 facilitates HCC metastasis by inducing bone marrow-derived cells recruitment into the metastatic site ${ }^{[23]}$. These results indicated that LOXL2 inhibition could reduce the proliferation and expansion of LCSCs. Targeting LCSCs by LOXL2 inhibitors may become an attractive and novel therapeutic strategy in HCC. 
We tried to explore the potential mechanisms of LOXL2 inhibiting LCSCs function. In the current study, the growth of CD133+ HepG2 and CD133 ${ }^{+} \mathrm{Hep} 3 \mathrm{~B}$ cells significantly slowed down after stable knockout of LOXL2. The CD133+Hep3B cell was even stagnant or dead. Apparently, knock down of LOXL2 may lead to LCSCs death via some mechanism. Cell death is subdivided into apoptosis, autophagy death and necrosis ${ }^{[28]}$. The activation of anti-apoptosis proteins benefits tumor cells from escaping apoptotic cell death ${ }^{[4,26]}$. We first investigated whether knock down of LOXL2 blunted the anti-apoptosis mechanism of LCSCs. We found that knock down of LOXL2 effectively downregulated the anti-apoptosis proteins BIRC3

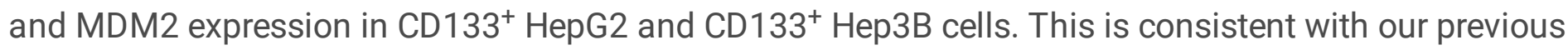
finding by microarray that BIRC3 and MDM2 are possible LOXL2 downstream genes in SMMC-7721 human hepatoblastoma cell lines ${ }^{[29]}$. BIRC3, one of the human inhibitor of apoptosis protein (IAP) family, is often overexpressed in HCC tissues and indicates a poor prognosis of HCC patients ${ }^{[30 ~ 32]}$. BIRC3 also induces EMT, proliferation, migration of hepatoblastoma cells in vitro ${ }^{[32]}$. Moreover, MDM2 is an upregulator of XIAP, which also belongs to the IAP family. It favors resistance of neoplastic cells to irradiation-induced apoptosis ${ }^{[33,34]}$. MDM2 is also a negative-regulatory factor of $p 53$, which is a central player in apoptosis ${ }^{[33,35 \sim 37]}$. We speculate that LOXL2 might benefit LCSCs from escaping apoptotic cell death by regulating BIRC3 and MDM2. Knockdown of LOXL2 could induce LCSCs apoptosis through downregulation of BIRC3 and MDM2, which may become a promising strategy of targeting LCSCs.

Another mechanisms of LOXL2 inhibiting LCSCs function is that LOXL2 might play a role in regulation of LCSCs autophagy. We investigated the expression change of autophagy marker LC3B and autophagy gene ATG5 upon LOXL2 gene knock down to understand whether LOXL2 was related with autophagic cell death. Our results show that knock down of LOXL2 downregulated the expression of LC3B and ATG5 in CD133 ${ }^{+}$HepG2 and CD133+Hep3B cells. This phenomenon could be explained by the previous reports that autophagy favors LCSCs resistance to hypoxia and nutrient deficiency in the tumor microenvironment of $\mathrm{HCC}{ }^{[38 \sim 40]}$. Autophagy positively regulates LCSCs by suppressing p53, which would normally be upregulated by mitochondrial PINK1 to downregulate CSC-related NANOG ${ }^{[41]}$. Moreover, BIRC3 was upregulated in the active autophagy response and inhibition of autophagy suppressed the expression of BIRC2 and BIRC3 ${ }^{[42,43]}$. Thus, we speculate that LOXL2 might take a part in regulation of LCSCs autophagy, favoring LCSCs survival under cellular stress and hypoxia.

However, the specific mechanism of LOXL2 in LCSCs is not well established. The relationship between LOXL2, apoptosis and autophagy need to be further explored. Moreover, BIRC3, MDM2 and other possible LOXL2 downstream genes need further investigations, such as p53 which is known associated with MDM2 and autophagy.

In summary, knock down of LOXL2 inhibits the proliferation, invasion and migration of LCSCs, and induces cell apoptosis and cell cycle arrest. Knockdown of LOXL2 effectively inhibited the expression of anti-apoptosis proteins BIRC3 and MDM2, as well as autophagy marker LC3B and autophagy gene ATG5 in LCSCs. LOXL2 inhibition could reduce the proliferation and expansion of LCSCs, making LOXL2 inhibitors an attractive and novel therapeutic strategy of HCC. 


\section{Statements And Declarations}

\section{Acknowledgments}

We would like to acknowledge support from the Laboratory of Integrated Traditional Chinese and Western Medicine of the First Affiliated Hospital of Dalian Medical University.

Funding: This work was supported by National Natural Science Foundation of China (No. 81673728).

Competing Interests: All authors have no conflicts of interest to declared.

Authors Contributions $\square Y Z$ conceived the work, and approved the final version of manuscript. Material preparation and experimental operation were performed by NL, HG, LL, XLZ and QLC. Data collection and analysis were performed by NL and HG. The manuscript was written by NL. All authors approved the final revision.

Ethics approval: The scheme of the current study was approved by the Institutional Review Board and Ethics Committee of the First Affiliated Hospital of Dalian Medical University. This study does not involve human or animal experiment.

Consent to participate: Not applicable.

Consent for publication: Not applicable.

\section{References}

[1] Laursen L (2014) A preventable cancer. Nature 516(7529): S2-3. doi: 10.1038/516S2a.

[2] Llovet JM, Zucman-Rossi J, Pikarsky E, et al (2016) Hepatocellular carcinoma. Nat Rev Dis Primers 2:16018.

[3] Nio K, Yamashita T, Kaneko S (2017) The evolving concept of liver cancer stem cells. Mol Cancer 16(4):4.

[4] Marquardt JU, Edlich F (2019) Predisposition to Apoptosis in Hepatocellular Carcinoma: From Mechanistic Insights to Therapeutic Strategies. Front Oncol 9:1421.

[5] Bruttel VS, Wischhusen J (2014) Cancer Stem Cell Immunology: Key to Understanding Tumorigenesis and Tumor Immune Escape? Front Immunol 5:360.

[6] Sistigu A, Musella M, Galassi C, et al (2020) Tuning Cancer Fate: Tumor Microenvironment's Role in Cancer Stem Cell Quiescence and Reawakening. Front Immunol 11:2166.

[7] Chan LH, Luk ST, Ma S (2015) Turning hepatic cancer stem cells inside out--a deeper understanding through multiple perspectives. Mol Cells 38(3):202. 
[8] Cheng Z, Li X, Ding J (2015) Characteristics of liver cancer stem cells and clinical correlations. Cancer Lett 379(2):230.

[9] Na Li, Ying Zhu (2019) Targeting liver cancer stem cells for the treatment of hepatocellular carcinoma. Ther Adv Gastroenterol 12:1-22.

[10] Liu L L, Fu D, Ma Y, et al (2011) The Power and the Promise of Liver Cancer Stem Cell Markers. Stem Cell Develop 20(12):2023-2030.

[11] Liu Y M, Li XF, Liu H, et al (2015) Ultrasound-targeted microbubble destruction-mediated downregulation of CD133 inhibits epithelial-mesenchymal transition, stemness and migratory ability of liver cancer stem cells. Oncol Rep 34(6):2977.

[12] Stephan M, Manish S, Alf S, Julio V, Pützer Brigitte M (2018) Emerging functional markers for cancer stem cell-based therapies: Understanding signaling networks for targeting metastasis. Semin Cancer Biol 53: S1044579X1830018X-.

[13] Cristóbal Ion, Rincón Raúl, Rebeca M, Federico R, García-Foncillas Jesús (2014) Role of oncogenic kras in cancer stem cell activation by aberrant wnt/ $\beta$-catenin signaling. J Natl Cancer Inst 8: djt373.

[14] L Ciuffreda, I Falcone, UC Incani, A Del Curatolo, F Conciatori, S Matteoni, S Vari, V Vaccaro, F Cognetti, M Milella (2014) PTEN expression and function in adult cancer stem cells and prospects for therapeutic targeting. Adv Biol Regul 56;66-80.

[15] Csiszar K (2001) Lysyl oxidases: a novel multifunctional amine oxidase family Prog Nucleic Acid Res Mol Biol 70:1-32.

[16] Vallet SD, Ricard-Blum S (2019) Lysyl oxidases: from enzyme activity to extracellular matrix crosslinks. Essays Biochem 63(3):349-364.

[17] Lucero HA, Kagan HM (2006) Lysyl oxidase: an oxidative enzyme and effector of cell function. Cell Mol Life Sci 63(19-20):2304-2316.

[18] Akiri G, Sabo E, Dafni H, et al (2003) Lysyl oxidase-related protein-1 promotes tumor fibrosis and tumor progression in vivo. Cancer Res Apr 1;63(7):1657-66. Erratum in: Cancer Res. 2004 Mar 15;64(6):2306. PMID: 12670920.

[19] Peinado H, Moreno-Bueno G, Hardisson D, et al (2008) Lysyl oxidase-like 2 as a new poor prognosis marker of squamous cell carcinomas. Cancer Res 68:4541-4550.

[20] Martin A, Salvador F, Moreno-Bueno G, Floristan A, Ruiz-Herguido C, Cuevas EP (2015) Lysyl oxidaselike 2 represses Notch1 expression in the skin to promote squamous cell carcinoma progression. EMBO $J$ 34(8):1090-1109. 
[21] Hong X, Yu J (2019) Silencing of lysyl oxidase-like 2 inhibits the migration, invasion and epithelial-to-mesenchymal transition of renal cell carcinoma cells through the Src/FAK signaling pathway. Int J Oncol 54(5):1676-1690.

[22] Cuevas EP, Eraso P, MJ Mazón, et al (2017) LOXL2 drives epithelial-mesenchymal transition via activation of IRE1-XBP1 signalling pathway. Sci Rep 7:44988.

[23] Carmen C, Aki P W, Huang Y P, et al (2014) Lysyl oxidase-like 2 is critical to tumor microenvironment and metastatic niche formation in hepatocellular carcinoma. Hepatology 60(5):1645-1658.

[24] Wu L, Zhu Y (2015) The function and mechanisms of action of LOXL2 in cancer. Int J Mol Med 36(5):1200-4.

[25] Liang C, Wang X, Zhang Z, et al (2020) ACOT11 promotes cell proliferation, migration and invasion in lung adenocarcinoma. Transl Lung Cancer Res 9(5):1885-1903.

[26] Saleem M, Qadir MI, Perveen N, et al (2013) Inhibitors of Apoptotic Proteins: New Targets for Anticancer Therapy. Chem Biol Drug Design 82(3):243-251.

[27] Lee TKW, Cheung VCH, Ng IOL (2013) Liver tumor-initiating cells as a therapeutic target for hepatocellular carcinoma. Cancer Lett 338(1):101-109.

[28] Booth LA, Tavallai S, Hamed HA, Cruickshanks N, Dent P (2014) The role of cell signalling in the crosstalk between autophagy and apoptosis. Cell Signal Mar;26(3):549-55.

[29] Wu L H, Zhang Y, Zhu Y, et al (2015) Mechanism Analyses for Elucidating the Role of LOXL2 knockdown in Hepatocellular Carcinoma. J Agr Sci Tech 5(5):370-379.

[30] Frazzi R (2021) BIRC3 and BIRC5: multi-faceted inhibitors in cancer. Cell Biosci Jan 7;11(1):8. doi: 10.1186/s13578-020-00521-0.

[31] Smolewski P, Robak T (2011) Inhibitors of apoptosis proteins (IAPs) as potential molecular targets for therapy of hematological malignancies. Curr Mol Med 11(8):633-649.

[32] Fu PY, Hu B, Ma XL, et al (2019) New insight into BIRC3: A novel prognostic indicator and a potential therapeutic target for liver cancer. J Cell Biochem 120(1):6035-6045.

[33] Arkin M (2005) Protein-protein interactions and cancer: small molecules going in for the kill. Curr Op Chem Biol 9(3):317-324.

[34] Gu L, Zhu N, Zhang H, et al (2009) Regulation of XIAP translation and induction by MDM2 following irradiation. Can Cell 15:363-375.

[35] Moreno-Càceres, Joaquim, Fabregat I (2015) Apoptosis in liver carcinogenesis and chemotherapy. Hepat Oncol 2(4):381-397. 
[36] Zhao Y, Yu H, Hu W (2014) The regulation of MDM2 oncogene and its impact on human cancers. Acta Biochim Biophys Sin 46(3):180-189.

[37] Cao H, Chen X, Wang Z, et al (2020) The role of MDM2-p53 axis dysfunction in the hepatocellular carcinoma transformation. Cell Death Discov 6:1-4.

[38] Galluzzi, L, Pietrocola, F, Pedro JMB, Amaravadi, RK, Baehrecke, EH, Cecconi F, et al (2015) Autophagy in malignant transformation and cancer progression. EMBO J 34(7):856-80.

[39] Song YJ, Zhang SS, Guo XL (2013) Autophagy contributes to the survival of CD133+ liver cancer stem cells in the hypoxic and nutrient-deprived tumor microenvironment. Cancer Lett 339(1):70-81.

[40] Li J, Hu SB, Wang LY (2017) Autophagy-dependent generation of Axin2 ${ }^{+}$cancer stem-like cells promotes hepatocarcinogenesis in liver cirrhosis. Oncogene 36(48).

[41] Liu K, Lee J, Kim JY, et al (2017) Mitophagy Controls the Activities of Tumor Suppressor p53 to Regulate Hepatic Cancer Stem Cells. Mol Cell 68(2):281.

[42] He W, Wang Q, Xu J, et al (2012) Attenuation of TNFSF10/TRAIL-induced apoptosis by an autophagic survival pathway involving TRAF2- and RIPK1/RIP1-mediated MAPK8/JNK activation. Autophagy 8(12):1811-1821.

[43] Comb WC, Cogswell P, Sitcheran R, et al (2011) IKK-dependent, NF-KB-independent control of autophagic gene expression. Oncogene 30(14):1727.

\section{Figures}


A
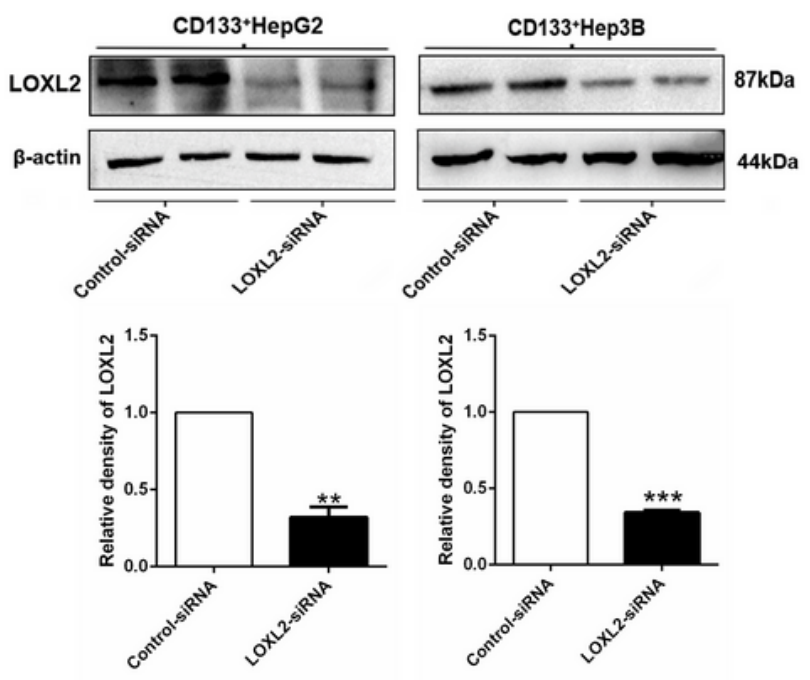

B

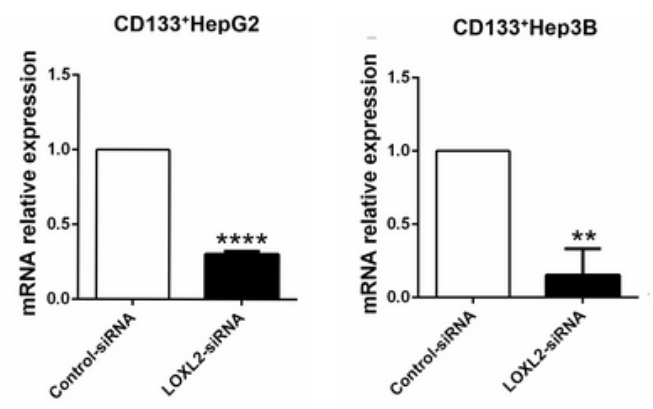

C
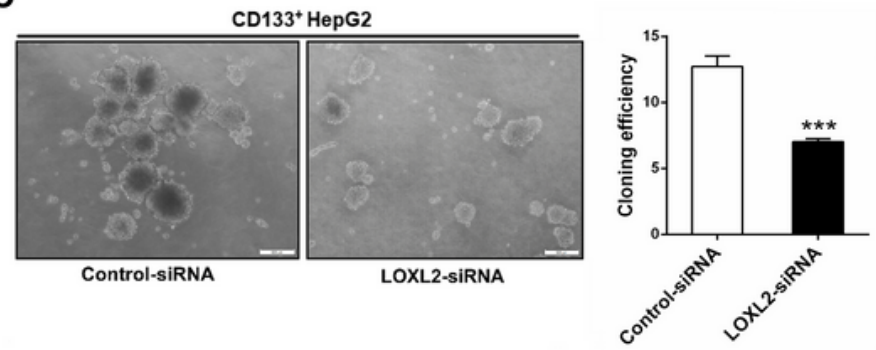

D
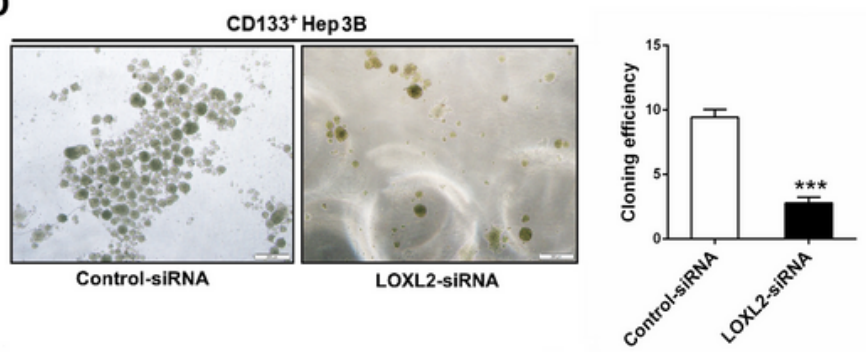

Figure 1

Knock down of lysyl oxidase-like 2 (LOXL2) impairs the spheroid formation of liver cancer stem cells (LCSCs) (A) Western blotting and (B) PCR were performed to determine transfection efficiency of LOXL2 gene in CD133+ HepG2 and CD133+ Hep3B cell lines 72 48h after LOXL2 or control siRNA lentiviral vectors were transferred. Relative intensity values for the proteins were obtained using Image J software. LOXL2 was effectively knocked down in CD133+ HepG2 and CD133+ Hep3B cell lines. Spheroid 
formation assay were performed to determine the effect of LOXL2 knockdown on cell survival in (C) CD133+ HepG2 and (D) CD133+ Hep3B cells. Knock down of LOXL2 impairs the spheroid formation of LCSCs. The data were analyzed upon three independent experiments and shown as mean \pm S.D. ${ }^{*} \mathrm{p}<$ $0.05,{ }^{* \star} p<0.01, * \star \star p<0.001, * \star \star \star x p<0.0001$.

A

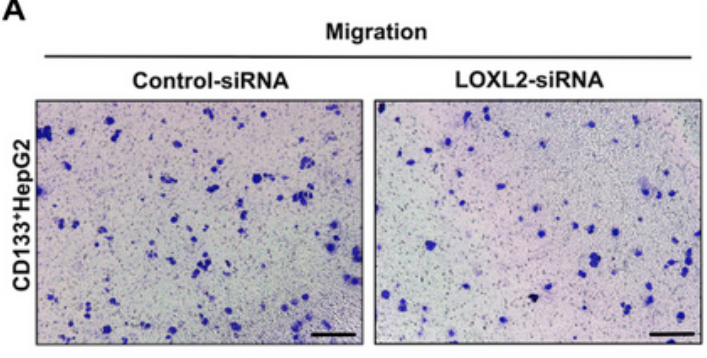

B

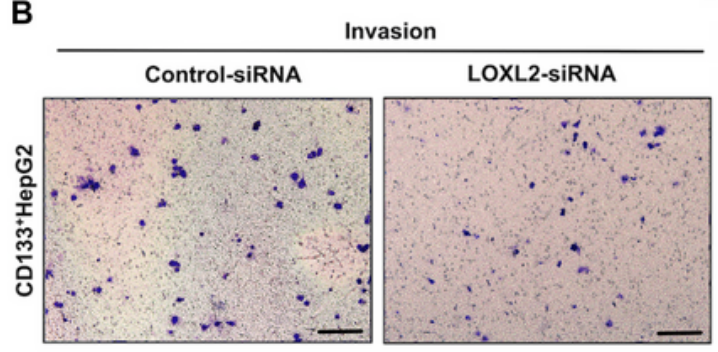

C

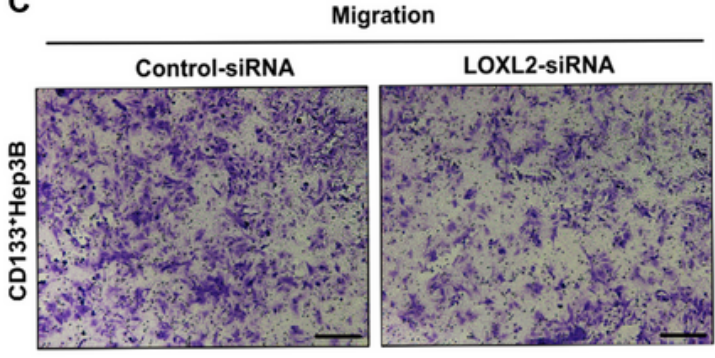

D

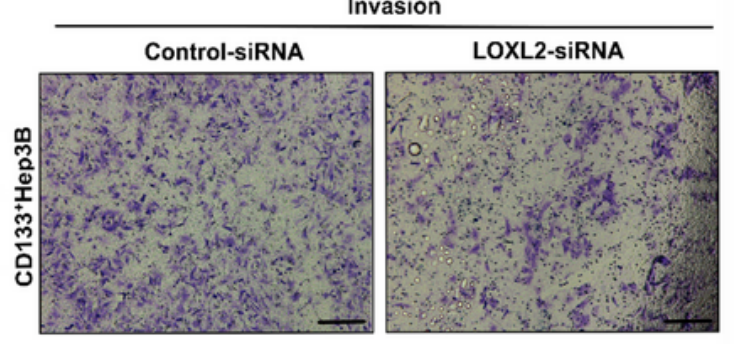

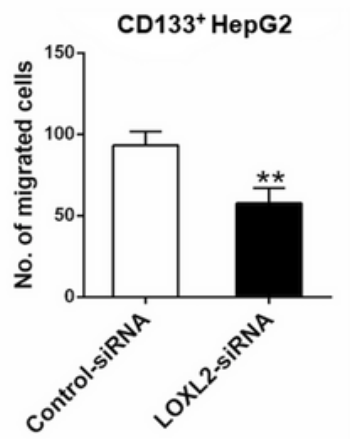

CD133+ HepG2

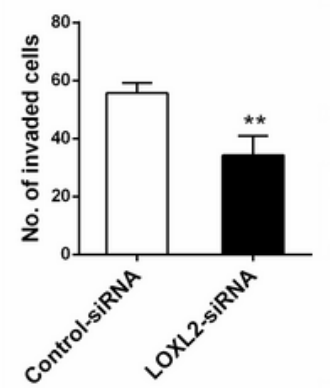

CD133+Hep3B

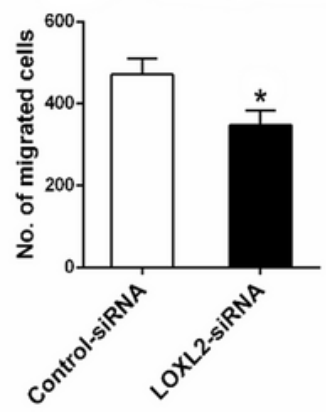

CD133Hep3B

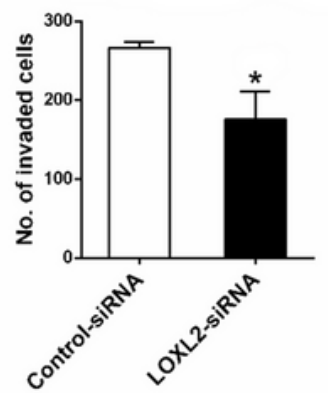

Figure 2 
Knock down of lysyl oxidase-like 2 (LOXL2) inhibits LCSCs migration and invasion (A, C) The transwell migration assays were performed upon LOXL2 knockdown in CD133+HepG2 and CD133+Hep3B cells. The average number of migrating cells are showed in graphs. (Scale bar: $50 \mu \mathrm{m}$ ). Knock down of LOXL2 inhibits LCSCs migration. (B, D) The transwell invasion assays were performed upon LOXL2 knockdown in CD133+HepG2 and CD133+Hep3B cells. The average number of invading cells are showed in graphs. (Scale bar: $50 \mu \mathrm{m}$ ). Knock down of LOXL2 inhibits LCSCs invasion. The data were analyzed upon three independent experiments and shown as mean \pm S.D. ${ }^{\star} p<0.05$, $* * p<0.01$.

A

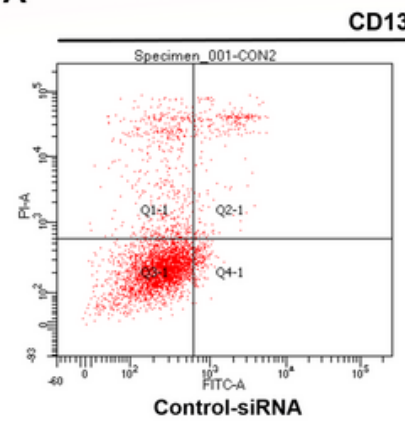

CD133+HepG2

B
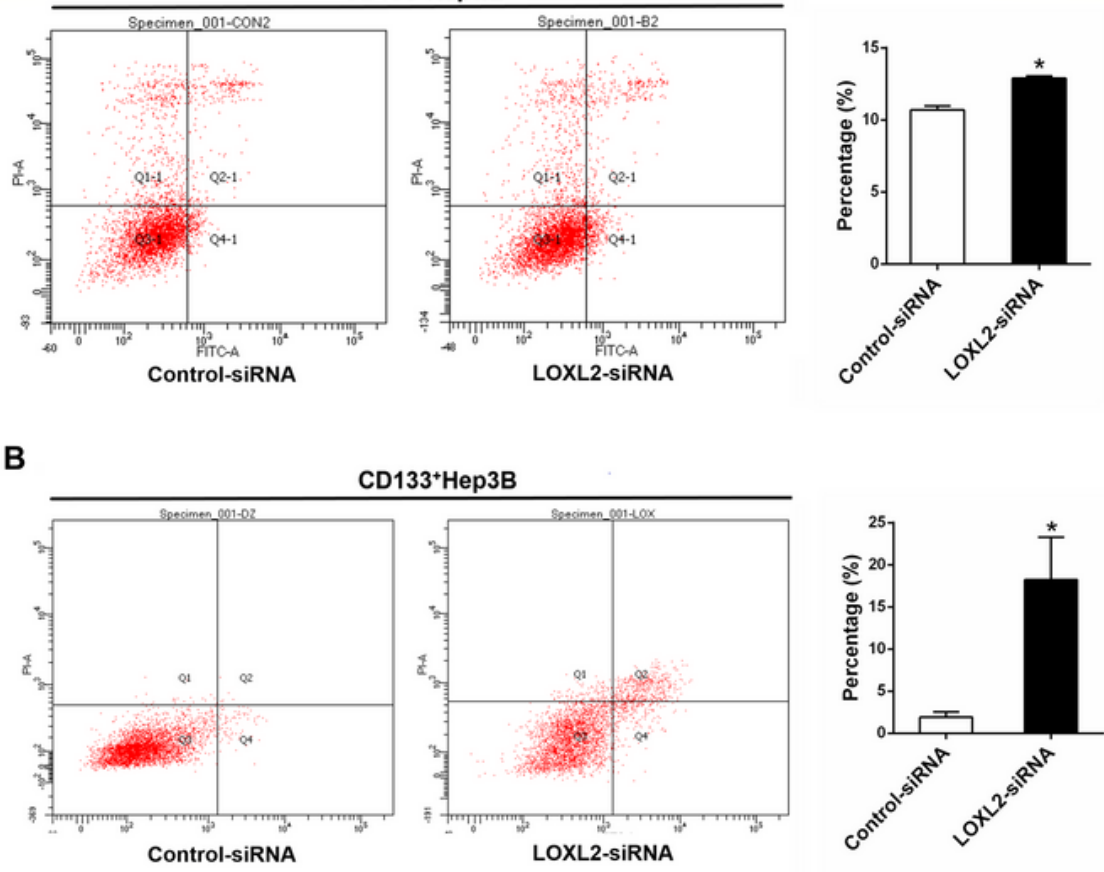

CD133+Hep3B

C
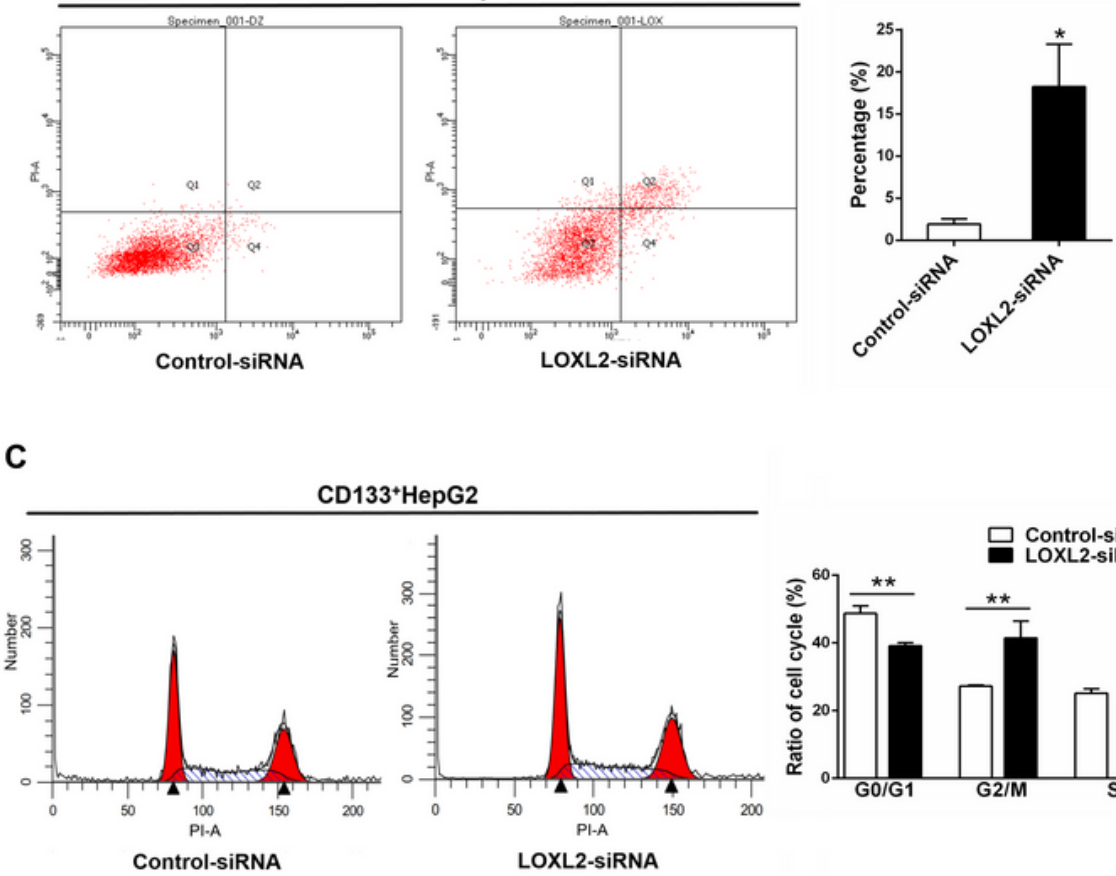

C133+HepG2

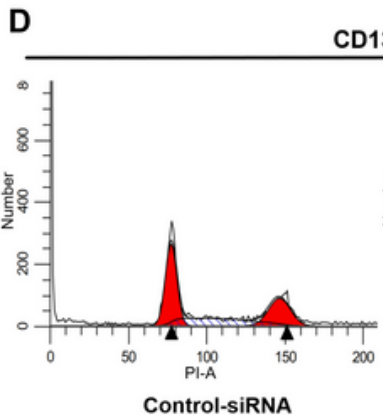

CD133Hep3B
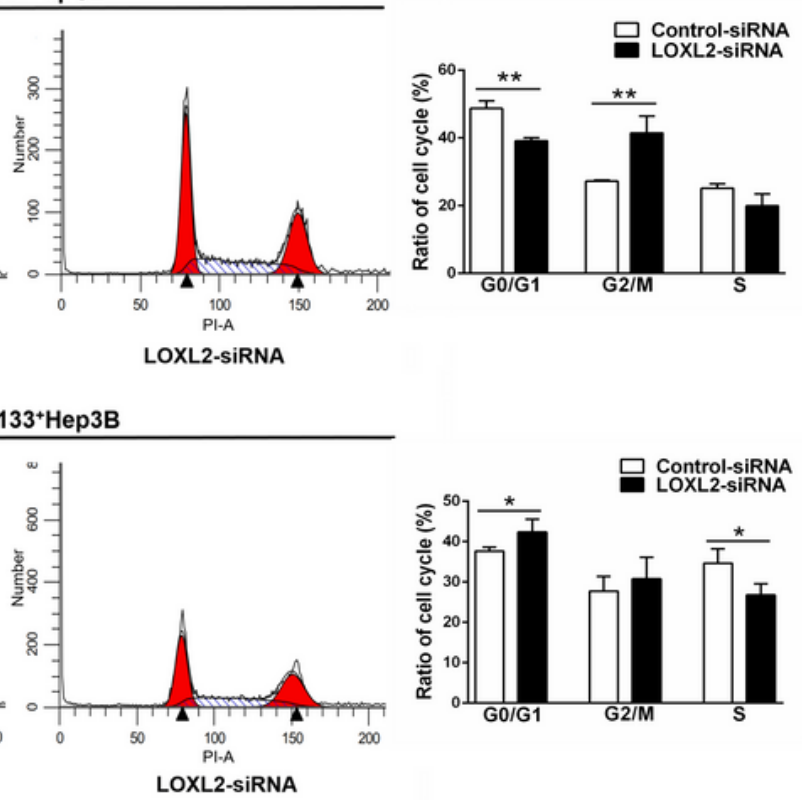

LOXL2-SIRNA

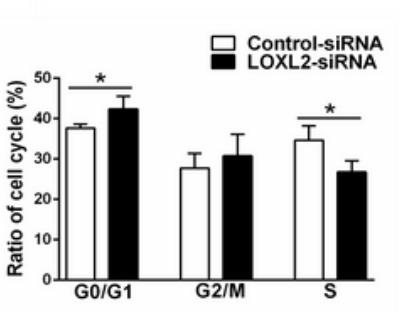




\section{Figure 3}

Knock down of lysyl oxidase-like 2 (LOXL2) induces apoptosis and cell cycle arrest in liver cancer stem cells (LCSCs) Fluorescence activated cell sorting (FACS) analysis showing apoptosis of CD133+ (A) HepG2 and (B) Hep3B cells transfected with control siRNA or LOXL2 siRNA. Cells were gated according to Annexin V-FITC and PI staining. Knock down of LOXL2 induces the apoptosis of LCSCs. Cell cycle assay of CD133+ (C) HepG2 and (D) Hep3B cells performed by flow cytometry upon LOXL2 knockout. Knock down of LOXL2 makes CD133+ HepG2 cell cycle arrest in G2/M phase, and CD133+Hep3B cells arrest in G0/G1 phase. Data represented as \% of total cells. The data were analyzed upon three independent experiments and presented as mean \pm S.D. ${ }^{*} p<0.05,{ }^{\star *} p<0.01$. 
A

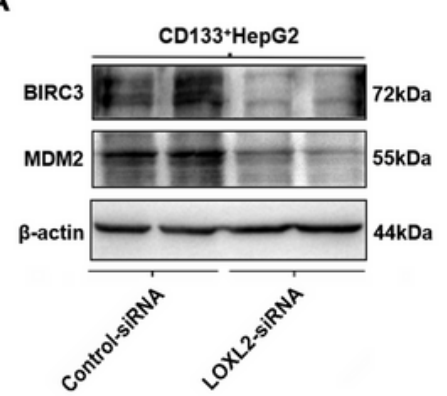

C
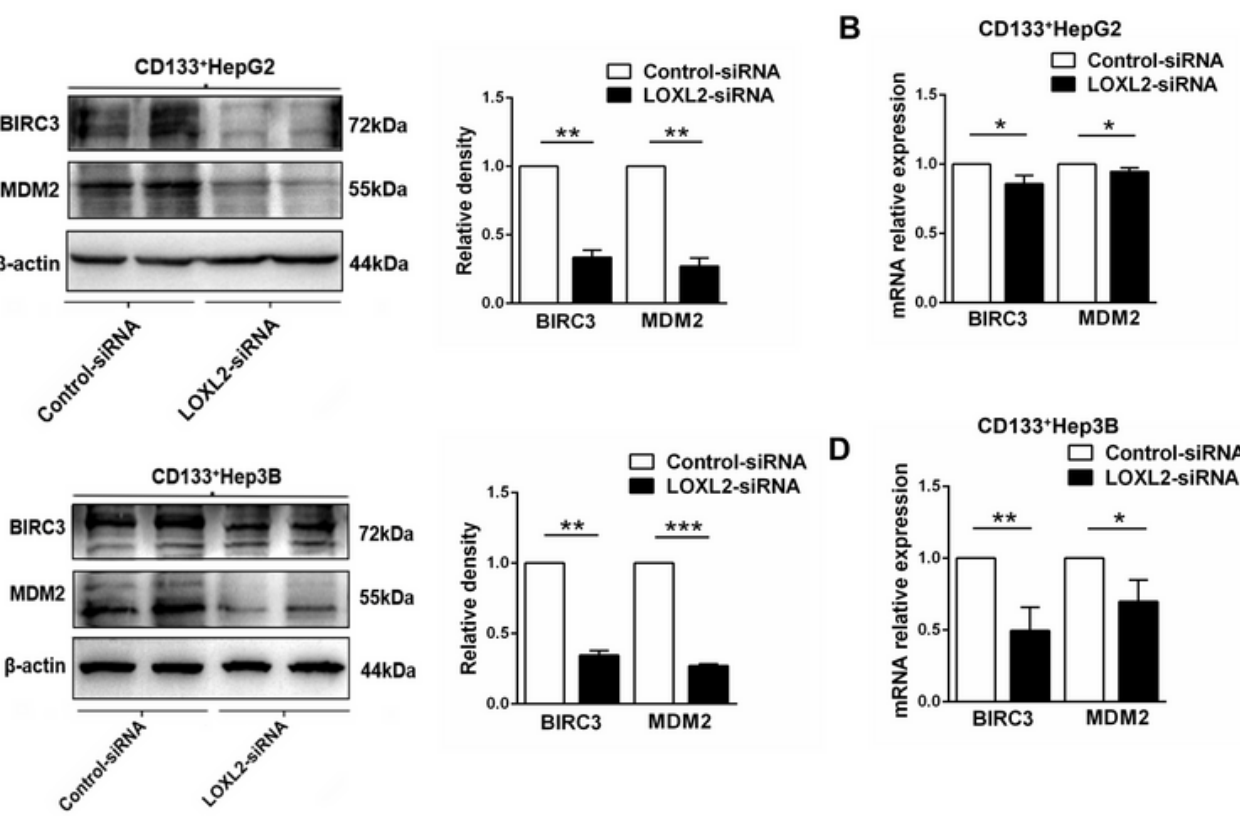

E
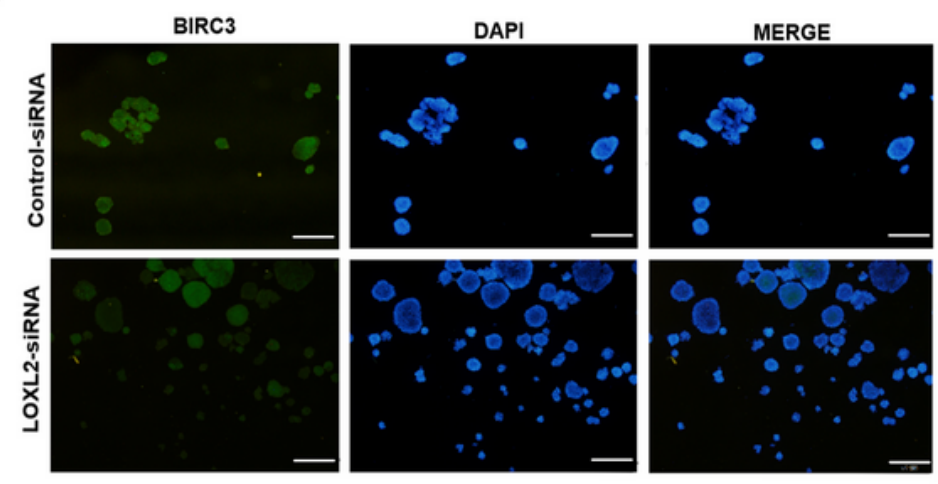

$\mathbf{F}$
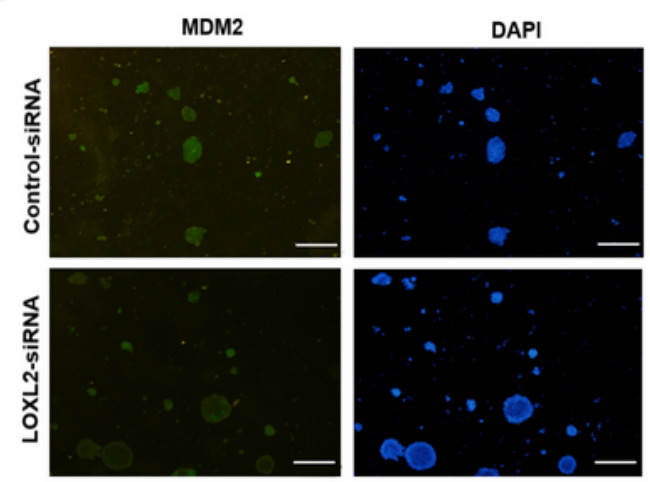
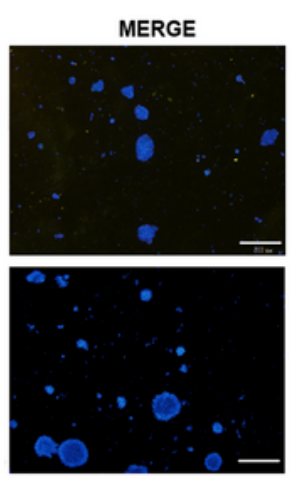
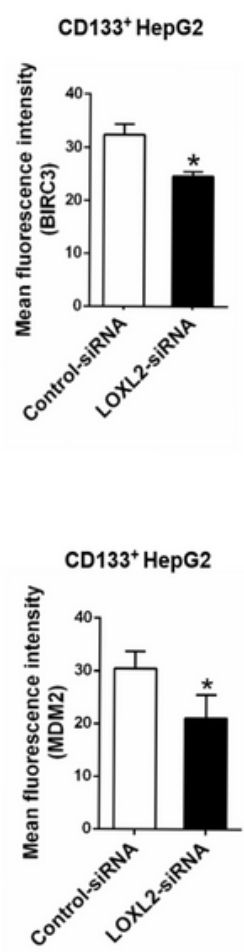

\section{Figure 4}

Knock down of Lysyl oxidase-like 2 (LOXL2) downregulates the anti-apoptosis proteins baculoviral IAP repeat-containing 3 (BIRC3) and murine double minute 2 (MDM2) in liver cancer stem cells (LCSCs) Western blotting and PCR were performed to determine the expression of BIRC3 and MDM2 in CD133+ (A, B) HepG2 and (C, D) Hep3B cells transfected with control siRNA or LOXL2 siRNA. Relative intensity values for the proteins were obtained using Image J software. (E, F) Immunofluorescence staining was 
performed for BIRC3 and MDM2 in CD133+ HepG2 cells upon LOXL2 knock down. Scale bars $200 \mu \mathrm{M}$. Mean fluorescence intensity values were obtained using Image $\mathrm{J}$ software. The data were analyzed upon three independent experiments. ${ }^{*} p<0.05,{ }^{*} p<0.01 .{ }^{* \star *} p<0.001$.

A

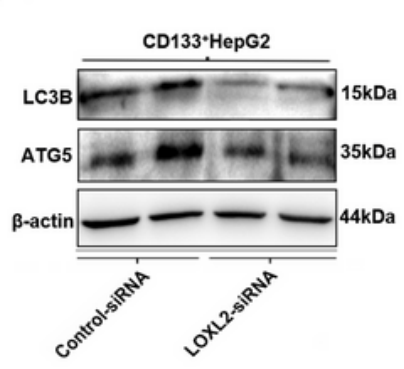

C

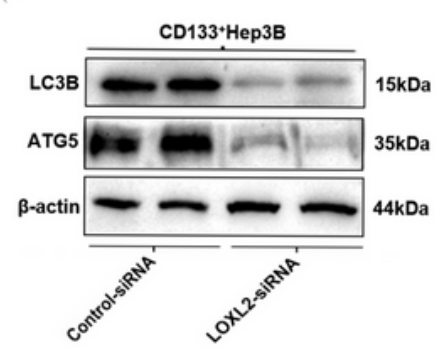

E
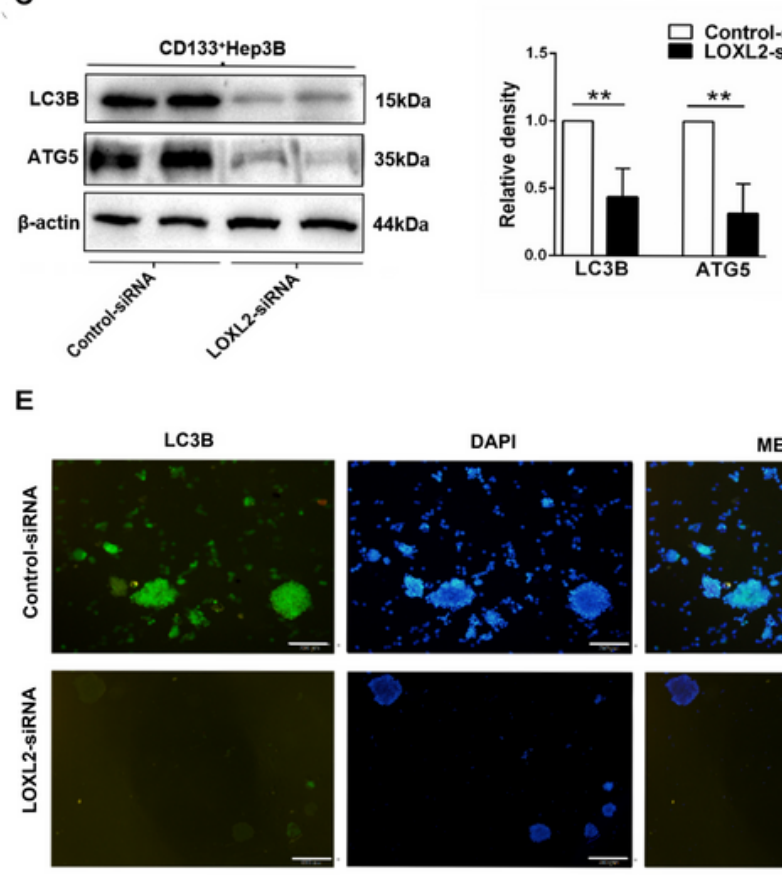

D
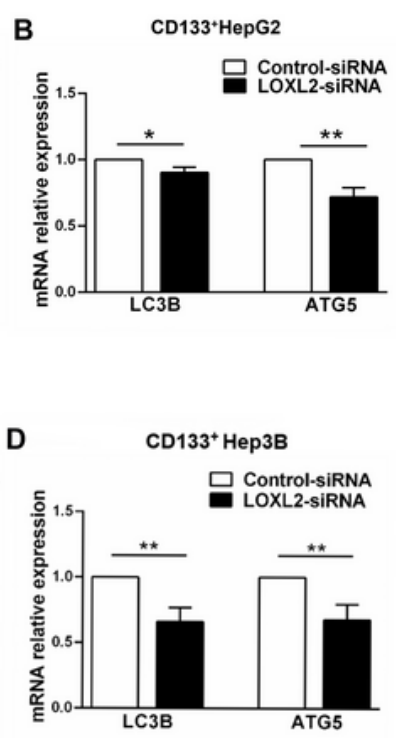

$\mathbf{F}$
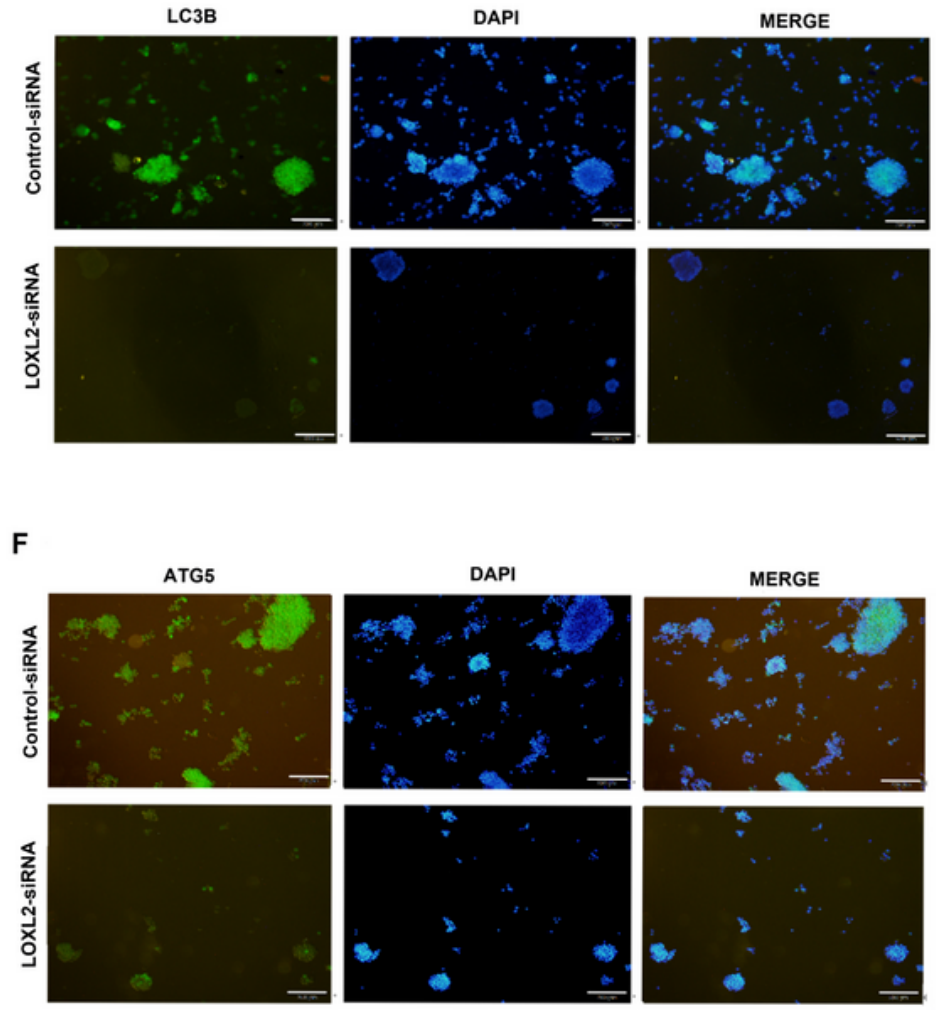
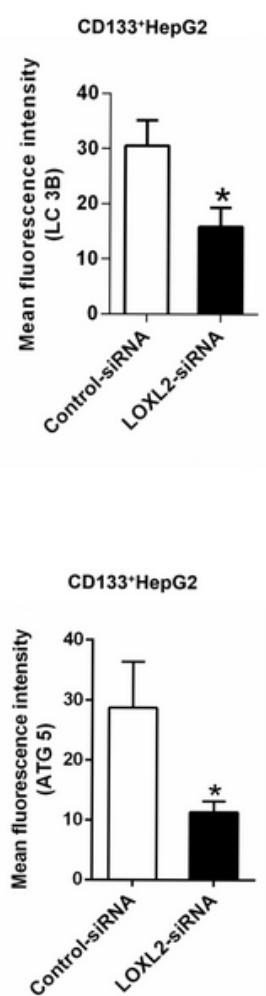

Figure 5

Knock down of Lysyl oxidase-like 2 (LOXL2) inhibits the expression of autophagy marker LC3B and autophagy gene ATG5 in liver cancer stem cells (LCSCs) Western blotting and PCR were performed to 
determine the expression of the autophagy marker type $B$ microtubule-associated protein 1 light chain 3 (LC3B) and autophagy gene ATG5 in CD133+ (A, B) HepG2 and (C, D) Hep3B cells transfected with control siRNA or LOXL2 siRNA. Relative intensity values for the proteins were obtained using Image $J$ software. (E, F) Immunofluorescence staining was performed for LC3B and ATG5 in CD133+ HepG2 cells upon LOXL2 knock down. Scale bars $200 \mu \mathrm{M}$. Mean fluorescence intensity values were obtained using Image $\mathrm{J}$ software. The data were analyzed upon three independent experiments. ${ }^{\star} p<0.05,{ }^{\star} * p<0.01$. 